\title{
High-resolution Panoramic Movie Generation from Video Streams Acquired by an Omnidirectional Multi-camera System
}

\author{
Sei IKEDA, Tomokazu SATO and Naokazu YOKOYA \\ Graduate School of Information Science, \\ Nara Institute of Science and Technology \\ 8916-5 Takayama, Ikoma, Nara, 630-0192 Japan \\ Email: $\{$ sei-i, tomoka-s, yokoya $\}$ is.aist-nara.ac.jp
}

\begin{abstract}
Telepresence systems using an omnidirectional image sensor enable us to experience remote sites with rich sensation. An omnidirectional multi-camera system is more useful to acquire high-resolution omnidirectional images of outdoor scenes than a monocular camera system. However, exact calibration of the camera system is necessary to generate a panoramic movie. In this paper, we describe a panoramic movie generation method based on geometric and photometric calibration of the omnidirectional multi-camera system. A prototype of telepresence system is also shown as applications of generated panoramic movies. This high-resolution telepresence system has been proven to enable us to experience remote sites with rich presence.
\end{abstract}

\section{Introduction}

Telepresence systems using an omnidirectional image sensor enable us to experience remote site. These systems are expected to be used in the fields of education and entertainment. A curved mirror [6] and a fish eye lens [7] have been widely used for these telepresence systems because they can be attached to a standard camera. However, these methods have problems of shortage and position-dependency of image resolution, because these sensors capture an omnidirectional scene by a single camera.

On the other hand, an omnidirectional multi-camera system can obtain a high-resolution image with uniform resolution, although exact camera calibration is necessary to generate a panoramic image from multiple images accurately. In related work, some calibration methods for omnidirectional multi-camera systems are proposed, which are based on using markers of known 3D positions[2]. In these methods, grid points of iterative patterns are used for markers and are projected on a large-sized immersive projection screen. However, it is difficult to obtain highly accurate calibration using such approaches, because the distribution of the markers is limited on a projection screen. Further more, there is no discussion about the precision of generated panoramic images.
In this paper, we propose a camera calibration and panoramic movie generation method for the omnidirectional multi-camera system "Ladybug". Figure 1 shows the multicamera system Ladybug made by Point Grey Research Inc. The camera unit consists of six cameras (Figure 1 (left)): Five configured in a horizontal ring and one pointing vertically. Figure 1 (right) shows a storage unit, which consists of four hard disks. The camera system can collect movies covering more than $75 \%$ of the full spherical view with almost the same apparent point of view.

This paper is constructed as follows. In Section 2, we describe a method of geometric and photometric calibration for the omnidirectional multi-camera system Ladybug. Section 3 describes a method of generating a spherical panoramic movie using the calibration result. The effect of disparity among multiple cameras is also discussed. In Section 4, the Ladybug camera system is actually calibrated geometrically and photometrically by using the proposed method. The spherical panoramic movie composed of six input images is also computed. A telepresence system is also prototyped in order to confirm that the panoramic movie can be used for telepresence well. In addition, we evaluate the discontinuity in computed panoramic images.

\section{Camera Calibration for Omnidirectional Multi-camera System}

This section describes a method of geometric and photometric calibration for an omnidirectional multi-camera system. In the geometric calibration, intrinsic and extrinsic parameters of each camera are estimated using a calibration board and a laser measurement system called total station. In the photometric calibration, limb darkening and color balances among multiple cameras are corrected.

\subsection{Geometric Calibration}

In the geometric calibration, intrinsic parameters (focal length $f$, lens distortion parameters $\left(\kappa_{1}, \kappa_{2}\right)$, center of distortion $\left(C_{x}, C_{y}\right)$, aspect ratio $\left.s_{x}\right)$ and extrinsic parameters (position $\boldsymbol{T}_{\boldsymbol{c}}$, orientation $\boldsymbol{R}_{\boldsymbol{c}}$ ) of each camera $c$ should be esti- 


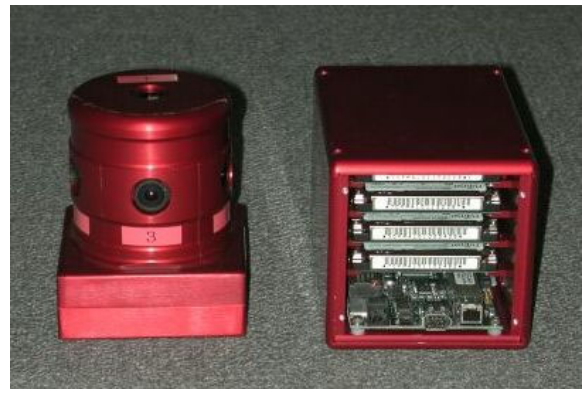

\section{Figure 1. Camera head (left) and storage unit (right) of Ladybug.}

mated. The extrinsic parameters are estimated in a unifiedcoordinate system to obtain the relationship among six cameras.

In camera calibration, spatial arrangement of many markers of known 3D position is required to estimate camera parameters accurately. First, arrangement of markers are described. In our method, grid points of a lattice pattern on a calibration board are used as markers. 3D coordinates of four corners of the calibration board are measured by the total station and all 3D positions of the markers on the board are calculated by linear interpolation among its corners. The calibration board is arranged and measured at several different positions for acquiring a large number of makers' 3D positions and 2D positions on images as shown in Figure 2. In our method, the total station must be fixed on the same position while the whole geometric calibration procedure is performed. Consequently, 3D positions of all markers captured by all the cameras can be represented in a single coordinate system made by the total station. Grid points on images are detected at sub-pixel resolution by calculating intersection of two lines.

Next, the camera parameters of each camera are estimated. The intrinsic parameters are estimated by Tsai's method[4]. In Tsai's method, the undistorted image coordinates $\left(X_{u}, Y_{u}\right)$ of markers are transformed to the distorted coordinates $\left(X_{d}, Y_{d}\right)$ by using following equations:

$$
\begin{gathered}
X_{u}=s_{x}\left(X_{d}-C_{x}\right)\left(1+\kappa_{1} r^{2}+\kappa_{2} r^{4}\right), \\
Y_{u}=\left(Y_{d}-C_{y}\right)\left(1+\kappa_{1} r^{2}+\kappa_{2} r^{4}\right), \\
r=\sqrt{X_{d}^{2}+Y_{d}^{2}} .
\end{gathered}
$$

Note that the distortion parameter $\kappa_{2}$ is also considered because input images from the cameras are highly distorted. The extrinsic parameters of each camera are estimated by minimizing the sum of re-projection errors $E_{c}$ of makers[5]. The re-projection error of a marker $m$ is defined as a squared distance on the image between the projected position $\boldsymbol{v}_{\boldsymbol{m}}$ of measured 3D position and the detected 2D position $\boldsymbol{u}_{\boldsymbol{m}}$ of the marker $m$. The measure $E_{c}$ is computed by:

$$
E_{c}=\sum_{m}\left(\boldsymbol{u}_{\boldsymbol{m}}-\boldsymbol{v}_{\boldsymbol{m}}\right)^{2}
$$

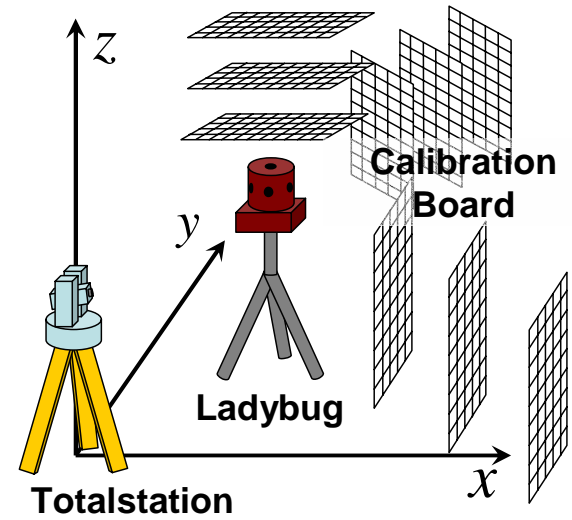

\section{Figure 2. Arrangement of calibration board in space.}

In this paper, extrinsic parameters of each camera are represented as a world-to-camera transformation matrix $M_{c}$ using position $\boldsymbol{T}_{\boldsymbol{c}}\left(t_{1}, t_{2}, t_{3}\right)$ and orientation $\boldsymbol{R}_{\boldsymbol{c}}\left(r_{1}, r_{2}, r_{3}\right)$ of each camera $c(c=1,2, \ldots, 6)$ on the world coordinate system:

$$
\begin{aligned}
\boldsymbol{M}_{\boldsymbol{c}}= & {\left[\begin{array}{cc}
\boldsymbol{R}_{\boldsymbol{c}} & \boldsymbol{T}_{\boldsymbol{c}} \\
0 & 1
\end{array}\right], } \\
= & {\left[\begin{array}{cccc}
c_{1} c_{3}+s_{1} s_{2} s_{3} & s_{1} c_{2} & -c_{1} s_{3}+s_{1} s_{2} c_{3} & t_{1} \\
-s_{1} c_{3}+c_{1} s_{2} s_{3} & c_{1} c_{2} & s_{1} s_{3}+c_{1} s_{2} c_{3} & t_{2} \\
c_{2} s_{3} & -s_{2} & c_{2} c_{3} & t_{3} \\
0 & 0 & 0 & 1
\end{array}\right] }
\end{aligned}
$$

where,

$$
\begin{aligned}
& s_{1}=\sin r_{1}, \quad s_{2}=\sin r_{2}, \quad s_{3}=\sin r_{3}, \\
& c_{1}=\cos r_{1}, \quad c_{2}=\cos r_{2}, \quad c_{3}=\cos r_{3} \text {. }
\end{aligned}
$$

Estimating $\boldsymbol{M}_{\boldsymbol{c}}$ for six parameters $\left(t_{1}, t_{2}, t_{3}, r_{1}, r_{2}, r_{3}\right)$ is a non-liner minimization problem. Since there exist problems concerning calculation cost and local minima, we first estimate $M_{c}^{\prime}$ for twelve parameters by minimizing $E_{c}$ linearly. Then, the estimated camera parameter $M_{c}^{\prime}$ is linearly adjusted to reduce the degree of freedom to six by assuming that the direction of optical axis is correctly estimated. Finally, the sum of re-projection error $E_{c}$ is minimized by a gradient method for optimizing $M_{\boldsymbol{c}}$.

\subsection{Photometric Calibration}

In the photometric calibration, first the limb darkening and color balances are corrected. First, the correction of the limb darkening is described. The limb darkening is a gradually decreasing effect of brightness in peripheral regions in images. Generally, images from an omnidirectional multi-camera system suffer from the limb darkening effect problem because wide-angle lenses are equipped. The $\cos ^{4} \theta$ phenomenon [1] and the vignetting [3] are well known as limb darkening effects. It is known that the $\cos ^{4} \theta$ phenomenon is proper to a wide-angle lens and the vignetting is proper to a zoom lens. 
Thus we only treat the $\cos ^{4} \theta$ phenomenon and correct image intensities using the following equation.

$$
I^{\prime}=\frac{\pi l^{2} \cos ^{4} \theta}{f^{2}} I,
$$

where $l$ is a lens diameter, $f$ is a focal length, $I$ is a radiance of surface of an object in the direction toward the lens, $I^{\prime}$ is an irradiance of the image and $\theta$ is an angle between the incident light and the optical axis.

Next, we describe a method for correcting color balances among cameras. Generally it is known that the irradiance of the image $I^{\prime}$ and the radiance of the surface $I$ have a linear relation shown as $I^{\prime}=a I+b$. The color balances are different due to the difference in color transform parameters $a$ and $b$ among cameras. In this paper, the color balance between two cameras $c$ and $c^{\prime}$ is adjusted by estimating parameters $a_{c}$ and $b_{c}$ in the linear transformation $I_{c^{\prime}}=a_{c} I_{c}+b_{c}$. The parameters $a_{c}$ and $b_{c}$ are determined by equalizing the normalized RGB histograms $h_{c}(i)$ and $h_{c^{\prime}}(i)$ where $i$ is intensity of an image pixel. The following error function $e\left(a_{c}, b_{c}\right)$ is minimized for equalizing the histograms $h_{c}(i)$ and $h_{c^{\prime}}(i)$ :

$$
e\left(a_{c}, b_{c}\right)=\sum_{i}\left\{h_{c^{\prime}}(i)-\frac{1}{a_{c}} h_{c}\left(\frac{i-b_{c}}{a_{c}}\right)\right\}^{2} .
$$

\section{Generating Omnidirectional Panorama Movie}

This section describes a method of generating omnidirectional panoramic images. The method is based on reprojecting calibrated input images on a spherical virtual image surface. We also discuss the effect of disparity between multiple cameras which do not satisfy the single viewpoint constraint.

\subsection{Generation of a Panorama Movie}

In generation of a panoramic image, limb darkening and the color balance of input images are corrected as a pretreatment, as shown in Figure 3. Then, corrected images are projected on a spherical projection surface by using the intrinsic and the extrinsic parameters of the geometric calibration result. In this section we describe a projection method of the corrected images.

Since the centers of projection of multiple cameras of the omnidirectional multi-camera system are different from each other, the single viewpoint perspective projection model is not applicable for this system. However, when the distance of a target from the system is sufficiently large, the centers of projection can be considered as the same. Therefore, we assume that the target scene is far enough from the system and set the spherical projection surface $S$ far enough from the camera system. The center of the spherical projection surface is set at a center of gravity of all the cameras' center of projection. A panoramic image is generated by projecting all the pixels of all the images onto the spherical surface $S$. Note that a blending method is used for generating a smooth panoramic image,

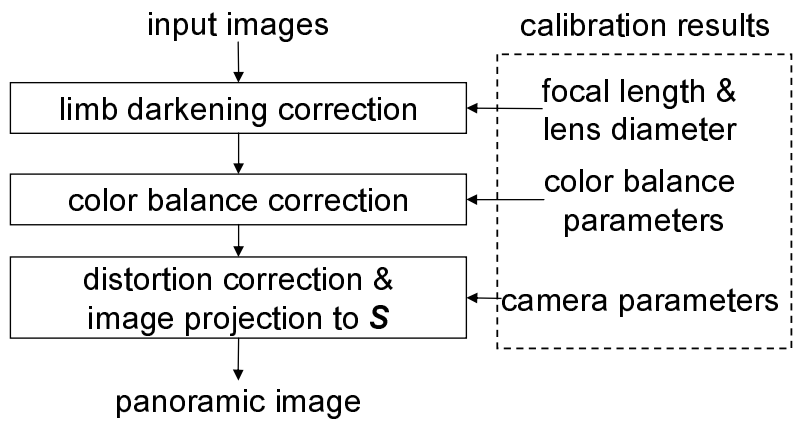

\section{Figure 3. Flow diagram of generating a panoramic image.}

when a point $s$ on the spherical surface $S$ is projected from image surfaces of different cameras. The intensity $I_{S}(s)$ of the point $s$ is determined by the following equation.

$$
I_{S}(s)=\frac{\sum_{c \in \boldsymbol{C}} \boldsymbol{C}_{(s)} \gamma_{c} I_{c}\left(u_{c}\right)}{\sum_{c \in \boldsymbol{C}(s)} \gamma_{c}},
$$

where $u_{c}$ is the point in the image of the camera $c$ which is projected on the point $s$ in the spherical surface $S, \gamma$ is the distance between $u_{c}$ and border of the image $c$, and $\boldsymbol{C}(s)$ is a set of cameras to which the point $s$ in the $S$ can be projected.

\subsection{Effect of Disparity}

We discuss the effect of disparity in camera position. As shown in Figure 4, when the target $x$ is captured at $u_{c}$ and $u_{c^{\prime}}$ on the images of the two cameras $c$ and $c^{\prime}$, they are projected to different position $s_{c}$ and $s_{c^{\prime}}$ on the spherical surface $S$ in the proposed method. Since $s_{c}$ and $s_{c}^{\prime}$ are not consistent, a panoramic image doubly shifted is generated. If the circumference of the spherical surface $S$ is composed of $N$ pixels, the effect of disparity is limited within one pixel if the condition $\angle s_{c} G s_{c^{\prime}}<\frac{2 \pi}{N}$ is satisfied. When the radius of the spherical surface $S$ is assumed to be infinite, $\angle s_{c} G s_{c^{\prime}}$ is approximated by $\angle s_{c} x s_{c^{\prime}}<\frac{2 \pi}{N}$. Consequently, the condition $\angle s_{c} G s_{c^{\prime}}<\frac{2 \pi}{N}$ is approximated by $\angle T_{c} x T_{c^{\prime}}<\frac{2 \pi}{N}$.

When we assume that the distance between the two cameras is $d$ and the distance from the each camera to the target $x$ is the same, the condition $\angle T_{c} x T_{c^{\prime}}<\frac{2 \pi}{N}$ is simplified that the distance $\lambda$ of the target $x$ from the center point of the two camera is represented as follows:

$$
\lambda>\frac{d}{2 \tan \frac{\pi}{N}} .
$$

\section{Experiment}

We have calibrated Ladybug system and have generated a panoramic movie. This section describes some experimental results concerning geometric and photometric calibration, panoramic movie generation, and telepresence application. 


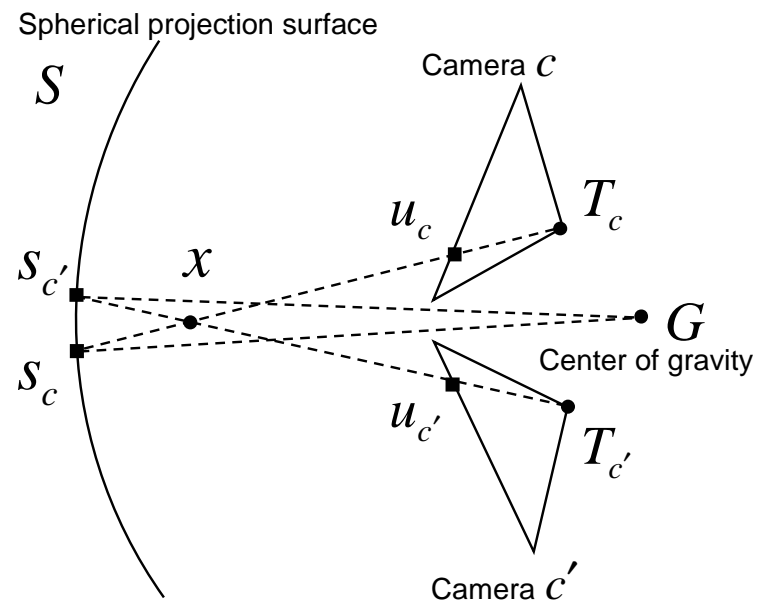

Figure 4. Effect of disparity.

\subsection{Calibration of Ladybug}

In the geometric calibration, the calibration board was captured by each camera of Ladybug fixed on a tripod. The calibration board was arranged and captured at different depth from fixed cameras three times. For each of five horizontal cameras, 187 grid points on the calibration board were used, then totally 561 set of 3D position and 2D position on captured images were obtained. For a vertical camera, 170 grid points on the calibration board were used, then totally 510 set of 3D position and 2D position on captured images were obtained. The 3D position was measured by a total station LEICA TCR1105 XR.

Figure 5 shows the result of reducing distortion of an input image using the estimated intrinsic parameters. We can confirm that the intrinsic parameters are fairly good. Figure 6 shows the result of correcting the limb darkening. The left is an input image. The right is a corrected image for the shortage of brightness in peripheral regions of the input image. Figure 7 shows a part of panoramic images before and after the color balance adjusting. In the right image, the boundary lines do not stand out compared with the non-corrected image in the left.

\subsection{Generation of a Panorama Movie}

A panoramic movie was generated by using the result of calibration described in the previous section. The input movie was obtained by setting Ladybug put on a moving car. Figure 8 shows an example set of input images (resolution: $768 \times 1024)$. A panoramic image (resolution: $3840 \times 1920$ ) generated from these input images is also shown in Figure 9. This panoramic image is unfolded by polar coordinates from the spherical surface. A black part at the bottom of the panoramic image is the part of no input images. Since any boundary lines are hardly found in Figure 9, we can confirm that geometrical and photometrical calibration is successfully achieved.

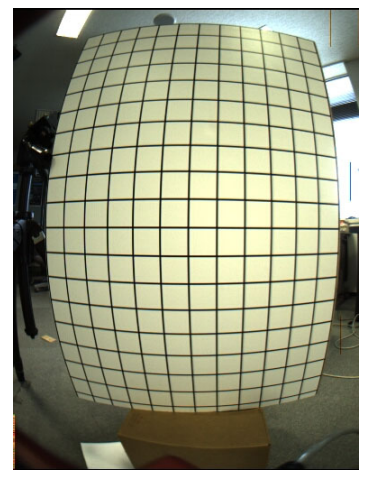

(a) input image

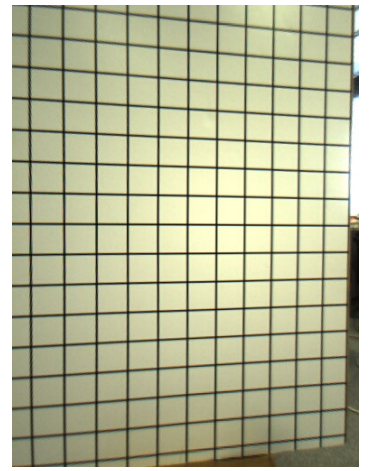

(b) corrected image
Figure 5. Lens distortion correction.

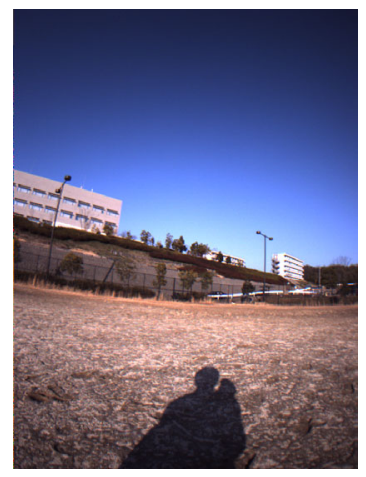

(a) input image

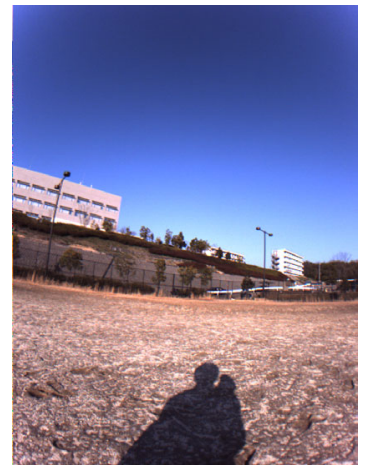

(b) corrected image
Figure 6. Limb darkening correction.

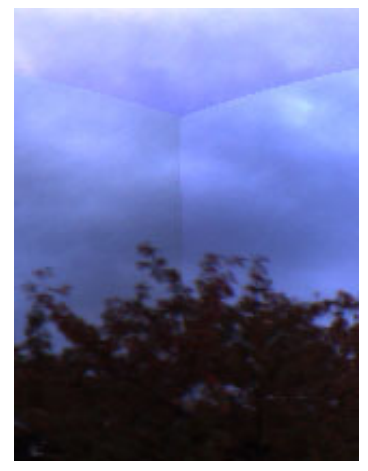

(a) non-corrected image

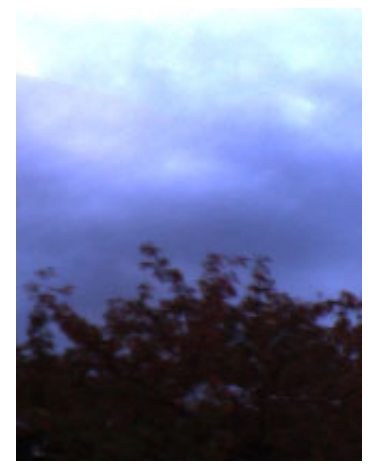

(b) corrected image
Figure 7. Color adjustment.

Figure 10 gives a panoramic image generated without the blend method blending for comparing it with the blended one. We can find some boundaries in the non-blended panoramic image; close objects and sky region containing saturated pixels due to direct rays of sun. On the other hand, we can not perceive any boundary lines in the blended panoramic image except very close objects. 


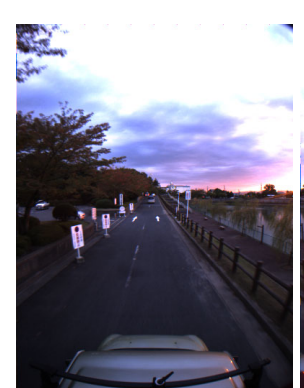

1

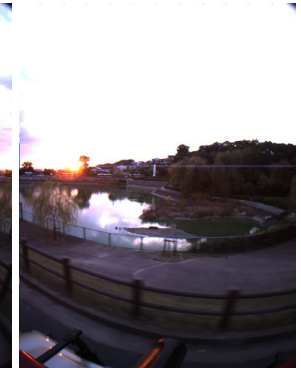

2

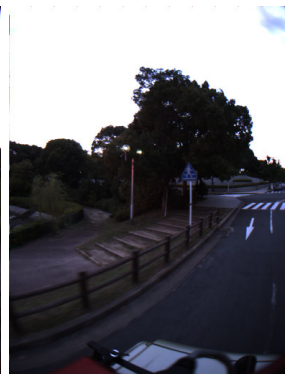

3

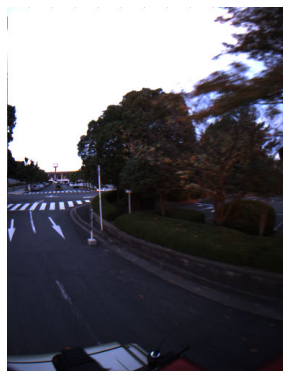

4

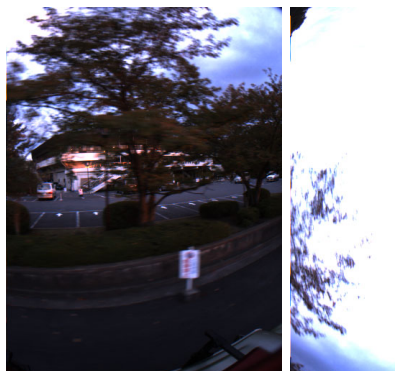

5

Figure 8. Input images: upper camera (right) and horizontal camera (others).

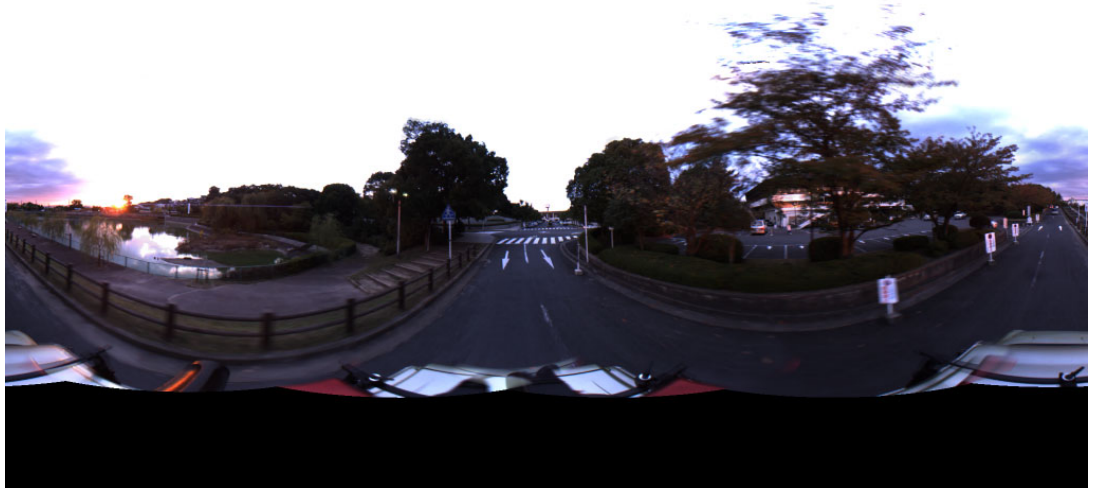

Figure 9. One frame of panoramic movie.

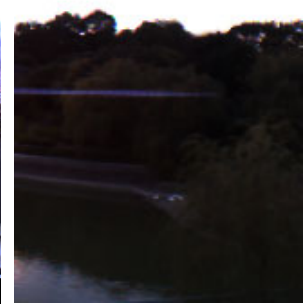

(a) blending

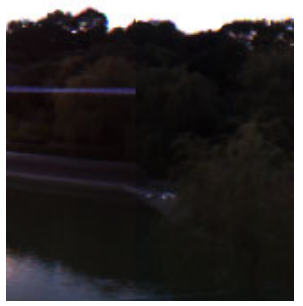

(b) no blending

Figure 10. Blending effect.

\subsection{Prototype Telepresence System}

A telepresence system was prototyped in order to confirm that the panoramic movie can be used for telepresence application well. The system is composed of a spherical immersive display, a controller and a personal computer as shown in Table 1. Our system enables users to interactively view an omnidirectional view field scene projecting a specified part of a panoramic movie in real time. The system performance of generating images is $20 \mathrm{fps}$, which is faster than the performance $15 \mathrm{fps}$ of image capturing by Ladybug. Due to the resolution limitation $1024 \times 768$ of the immersive display, the resolution of a panoramic movie was resized to $2048 \times 1024$ in the experiment. Figure 11 shows user's appearance in telepresence experiment. We have confirmed that our telepresence system exhibits high-presence in respect of resolution, field of view, and interaction.

Table 1. Components of telepresence system.

\begin{tabular}{lll}
\hline Spherical Display & Elumens VisionStaion & \\
Controller & Microsoft SideWinder Game Pad \\
& Pro & \\
PC & CPU:Intel Pentium4 & $1.7 \mathrm{GHz}$, \\
& Memory:1GB & \\
Graphics Card & Nvidia Geforce4 & \\
\hline
\end{tabular}

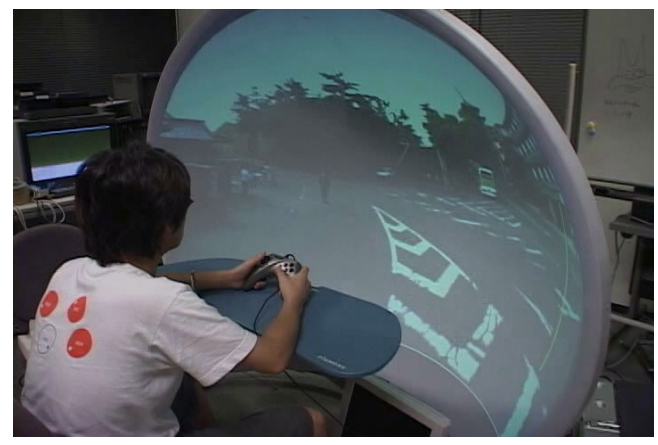

Figure 11. Appearance of telepresence system.

\subsection{Quantitative Evaluation}

We have evaluated errors in panoramic image generation. The error is defined as an angle between two lines connecting the spherical projection center $G$ and the two corresponding projected points of the same point in space on the spherical projection surface.

There are common regions in input images of adjacent cameras of Ladybug as shown in Figure 12. A circle marker is captured in these regions to specify the corresponding points of adjacent two camera images. The center of gravity of this 

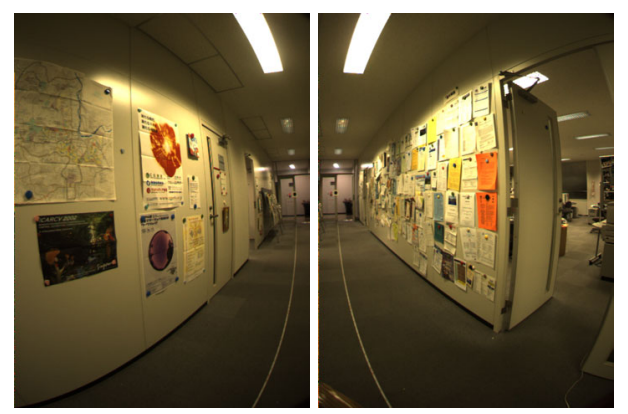

Figure 12. Common regions in input images.

marker is calculated with sub-pixel accuracy. In this experiment, the angle $\angle s_{c} G s_{c^{\prime}}$ shown in Figure 4 was evaluated as error $\epsilon$, where $u_{c}$ and $u_{c^{\prime}}$ are centers of gravity of these markers. Because the distance decreasing the effect of disparity within one pixel on the panoramic image is about $24 \mathrm{~m}$ under assumptions of $d=4 \mathrm{~cm}$ and $N=3840$, the marker was located about 30m away from Ladybug. More than one hundred corresponding points were obtained for each camera.

Table 2 shows maximum and average values of error $\epsilon$ in generation a panoramic image. The number 6 represents the vertical camera and the others represent horizontal cameras. The average value of the error $\epsilon$ is $0.0063 \mathrm{rad}$ which is approximately equivalent to 3 pixels on an input image. We have also found that points having large errors tend to distribute at the borders of input images.

\section{Conclusion}

In this paper, we have described a method of generating a high-resolution spherical panoramic movie based on the result of geometric and photometric calibration for an omnidirectional multi-camera system Ladybug. In the geometric calibration, a large number of markers were virtually arranged

\section{Table 2. Error $\epsilon$ of a generated panoramic image} [rad].

\begin{tabular}{ccccc}
\hline Camera Number & $1-2$ & $2-3$ & $3-4$ & $4-5$ \\
\hline Number of the data & 227 & 258 & 229 & 216 \\
Maximum value & 0.0214 & 0.0378 & 0.0258 & 0.0227 \\
Average value & 0.0072 & 0.0057 & 0.0054 & 0.0048 \\
\hline & & & & \\
\hline Camera Number & $5-1$ & $6-1$ & $6-2$ & $6-3$ \\
\hline Number of the data & 305 & 176 & 131 & 127 \\
Maximum value & 0.0198 & 0.0178 & 0.0498 & 0.0208 \\
Average value & 0.0041 & 0.0058 & 0.0144 & 0.0095 \\
\hline & & & & \\
\hline Camera Number & $6-4$ & $6-5$ & & \\
\hline Number of the data & 123 & 154 & & \\
Maximum value & 0.0107 & 0.0112 & & \\
Average value & 0.0063 & 0.0052 & & \\
\hline
\end{tabular}

around the system by using a calibration board and a total station for improving the accuracy of calibration. We have also prototyped a telepresence system having omnidirectional view of field. Experiments have exhibited that panoramic movies can be used for telepresence application. Finally, projected errors generating a panoramic image was evaluated. We have found that the average of the error is about 3 pixels on an input image. In future work, a better lens distortion model for wide-angle lens will be investigated for obtaining higher accuracy in generation of panoramic movie.

\section{References}

[1] B. K. P. Horn. Robot Vision :206-209. Mit Press, 1986.

[2] J. Shimamura, H. Takemura, N. Yokoya, and K. Yamazawa. Construction of an Immersive Mixed Environment Using an Omnidirectional Stereo Image Sensor. Proc. IEEE Workshop on Omnidirectional Vision :62-69, 2000.

[3] N. Asada, A. Amano, and M. Baba. Photometric Calibration of Zoom Lens Systems. Proc. Int. Conf. Pattern Recognition, A:186-190, 1996.

[4] R. Y. Tsai. A Versatile Camera Calibration Technique for Highaccuracy 3D Machine Vision Metrology Using Off-the-shelf TV Cameras and Lenses. IEEE Journal of Robotics and Automation, RA-3(4):323-344, 1987.

[5] T. Sato, M. Kanbara, N. Yokoya, and H. Takemura. Dense 3D Reconstruction of an Outdoor Scene by Hundreds-Baseline Stereo Using a Hand-Held Video Camera. Int. Journal of Computer Vision, 47(1-3):110-129, 2002.

[6] Y. Onoe, K. Yamazawa, H. Takemura, and N. Yokoya. Telepresence by Real-time View-dependent Image Generation from Omnidirectional Video Streams. Computer Vision and Image Understanding, 71(2):154-165, 1998.

[7] Z. L. Cao, S. J. Oh, and E. L. Hall. Dynamic Omnidirectional Vision for Mobile Robots. Journal Robotic Systems, 3(1):5-17, 1986. 